\title{
Tucker S. McElroy, Dimitris N. Politis (2020): Time series: a first course with bootstrap starter
}

\author{
CRC Press, Boca Raton, 586 pp. \$ 79.96 (Hardcover), ISBN \\ 978-1-4398-7651-0
}

\section{Marco Meyer ${ }^{1}$}

Received: 6 September 2020 / Revised: 15 September 2020 / Published online: 16 October 2020 (c) The Author(s) 2020

Modern Time Series Analysis has been an important branch within the field of statistics for about the last 100 years, and a considerable number of classical textbooks has been written about this subject. Some of these books dating back to the 1970s are still widely considered as valuable sources of information for researchers and lecturers alike. These monographs can mostly be divided into two groups. The first is primarily directed at practitioners and usually focuses on a description of various methods to work on time series data while providing merely basic introduction to the theoretical background (if at all). The second usually contains a sound mathematical introduction to the subject which requires knowledge in probability theory, particularly in measure theory. Some textbooks in this second group are therefore excellent choices for researchers and for instructors of $\mathrm{PhD}$ level courses on time series, while being hardly accessible for undergraduate students or practitioners.

This dilemma was the starting point for McElroy and Politis to design a textbook that fits right in between these two groups. As the authors put it in the Preface, "the challenge that we decided to undertake was to produce a text that satisfies the triptych: (i) mathematical completeness - albeit at a slightly lower level than [Brockwell and Davis (1991)], (ii) computational illustration and implementation, and (iii) conciseness and accessibility to upper-level undergraduate and M.S. students". The result is a book that clearly achieves this ambitious goal. Its more than 500 pages are well spent as this presentation of time series analysis is almost completely self-contained, solely requiring basic knowledge in mathematical statistics. The authors take their time to motivate and explain basic concepts in a way that in many instances does not require measure theory or complex analysis. It has to be emphasized that this kind of accessibility does not come at the price of a lack of mathematical depth; this is one notable achievement that sets this textbook apart. Especially in the sections about

Marco Meyer

marco.meyer@tu-bs.de

1 TU Braunschweig, Braunschweig, Germany 
spectral representation and entropy, some rather deep results are presented including their proofs which are left out in many other textbooks. Chapters and subsections with advanced content are clearly marked, and I particularly like the clear organization within the chapters. Each chapter ends with an overview which summarizes the most important concepts and "take home messages" from this section. This is followed up by a remarkable collection of exercises which are marked with different symbols as easy/difficult as well as theoretical/ computational. Also, almost all chapters contain examples about the implementation of the methods to real-world data with the software package $\mathrm{R}$ and corresponding exercises. The link between theory and practice is therefore a common thread that runs through the whole book.

The first four chapters include classical introductory topics starting with concrete examples of time series data and their graphical presentation. The fundamental concept of stationarity is introduced in Chap. 2, before the third chapter explains in detail how to deal with the typically observed deviations from stationarity in real-world time series. This is a topic that is in practice arguably the most important first step but that is somehow not given the attention it deserves in some other textbooks. Chapter 4 presents the connection of time series theory to Hilbert spaces and can be seen as a perfect example of how the authors achieve their aforementioned goal of "satisfying the triptych": This chapter is mathematically sound and complete while at the same time even readers with only basic mathematical background can grasp the connection to geometry through illustrations. However, this balancing act reaches its limits once conditional expectations are needed. McElroy/Politis refer to their Appendix where they develop the measure theoretic aspects while relying within the chapter on an intuitive understanding of these concepts. This is an elegant solution that stands exemplarily for the approach in this text.

Chapter 5 discusses the important class of ARMA models and Chaps. 6 and 7 are devoted to spectral theory. Splitting up the latter into two chapters works nicely: while Chap. 6 introduces very important concepts such as the spectral density and filtering in a way that is accessible for newcomers, Chap. 7 provides the deeper spectral theory. The connection of information theory to time series analysis is presented in Chap. 8, while Chaps. 9 and 10 are devoted to statistical aspects, model identification, and forecasting. The final Chaps. 11 and 12 are about two topics that could easily fill textbooks on their own: nonlinear time series and bootstrap methods. It is not within the scope of this book to go into all the details of these broad subjects, and the authors do a nice job of giving a concise overview-without getting too technical—and to motivate further reading on time series.

I can definitely recommend this book to students, lecturers and researchers alike. Whether one wants to design a course on time series for maths or statistics students or just for self-study: The authors of "Time Series - A First Course with Bootstrap Starter" provide clear paths for readers with different backgrounds how to use either the text as a whole or different portions thereof, and to work on one's own preferred level of mathematical depth.

Funding Open Access funding enabled and organized by Projekt DEAL. 
Open Access This article is licensed under a Creative Commons Attribution 4.0 International License, which permits use, sharing, adaptation, distribution and reproduction in any medium or format, as long as you give appropriate credit to the original author(s) and the source, provide a link to the Creative Commons licence, and indicate if changes were made. The images or other third party material in this article are included in the article's Creative Commons licence, unless indicated otherwise in a credit line to the material. If material is not included in the article's Creative Commons licence and your intended use is not permitted by statutory regulation or exceeds the permitted use, you will need to obtain permission directly from the copyright holder. To view a copy of this licence, visit http://creativecommons.org/licenses/by/4.0/.

\section{Reference}

Brockwell PJ, Davis RA (1991) Time series: theory and methods, 2nd edn. Springer, New York

Publisher's Note Springer Nature remains neutral with regard to jurisdictional claims in published maps and institutional affiliations. 